MATTERS ARISING

Complications of treatment for

The recent editorial on Palliation in $A I D S$ by George $^{1}$ and subsequent Clinico-pathological Conference on the Complications of treatment of cryptosporidial diarrhoe ${ }^{2}$ are timely reminders of the limitations and consequences of current AIDS therapy and the need to re-evaluate constantly the goals of AIDS management in each case.

In the Clinico-pathological Conference, bronchopneumonia and cardiac failure from staphylococcal septicaemia were found on necropsy to be the cause of death in a terminally-ill AIDS patient with a Hickman line and documented cryptosporidial diarrhoea. The prolonged presence of the indwelling line ( 2 months) and the isolation of Staphylococcus aureus from this site may have been factors for the later development of disseminated staphylococcal infection. ${ }^{3}$ However, staphylococcaemia is not recognised as a cell-mediated opportunist manifestation of AIDS, and its occurrence in this patient suggests an additional cofactor, possibly as a result of drug therapy.

It is now increasingly apparent that the use of myelotoxic drugs in AIDS patients is an important cause of neutropenia-associated opportunist infections. In particular, the antiviral agents zidovudine (AZT) and ganciclovir (DHPG) are known to cause neutropenia, ${ }^{45}$ which may result in opportunist infection with Staphylococcus aureus (as in this case), Klebsiella pnumoniae, E coli, Pseudomonas aeruginosa, other Enterobacteriaciae, as well as disseminated candidosis and aspergillosis. Staphylococcal infections are particularly relevant in this context, since AIDS patients are frequently subjected to a combination of myelotoxic agents and invasive procedures, such as insertion of Hickman lines. HIV infection in groups such as intravenous drug users are also likely to provide additional cases of disseminated staphylococcal infection. $^{6}$

Even if the neutrophil count was monitored in the final months of this patient's life, the palliative use of dexamethasone may have lead to impairment of neutrophil function, with the same consequences. Zidovudine was discontinued two months before the patient died, suggesting that this agent was probably not contributing to the pathological process.

With the increasing use of myelosuppressive drugs in HIV patients, physicians caring for these individuals should be vigilant to the risk of iatrogenic factors changing the patterns of disease presentation, and anticipate the additional problems that may arise as a consequence of neutropenia-associated opportunist infections.

Ludwig Institute for Cancer Research, St Mary's Hospital Medical School, Norfolk Place,

London W2 1PG, UK

1 George RJD. Palliation in AIDSwhere do we draw the line? Genitourin Med 1991;67:85-6.

2 Anderson J, George RJD, Weller IVD, Lucas SB, Miller RF. Complications of treatment for cryptosporidial diarrhoea. Genitourin Med 1991;67: 156-61.

3 Krumholz HM, Sande MA, Lo B. Community-acquired bacteremia in patients with acquired immunodeficiency syndrome: clinical presentation, bacteriology, and outcome. $\mathrm{Am}$ J Med 1989;86:776-9.

4 Schrager LK. Bacterial infections in AIDS patients. AIDS 1988;2(supp 1): $S 183-S 189$.

5 Chaisson RE. Infections due to encapsulated bacteria, salmonella, shigella and campylobacter. Infect Dis Clin North Am 1988;2:475-84.

6 Witt DJ, Craven DE, McCabe WR. Bacterial infections in adult patients with the acquired immune deficiency syndrome (AIDS) and AIDS-related complex. Am J Med 1987;82:900-6.

\section{Cervical infection with chlamydia trachomatis}

With great interest, I note two recent splendid publications by Radcliffe $e t$ al. ${ }^{12}$ The theme of both of these articles addresses the practice of obtaining a post treatment test of cure for endocervical chlamydial infection. I agree that test of cure is generally unnecessary following patient compliant therapy. Also, reinfection may be rare during the immediate post treatment period.

Our prospective follow up chlamydial test of cure publication ${ }^{3}$ may be relevant and noteworthy to substantiate several comments. We serially obtained three Chlamydia trachomatis cultures and three enzyme immunoassay (chlamydiazyme) tests from sixty-four patients treated for $C$ trachomatis cervicitis with tetracycline. All organisms were non viable

\section{Test of Cure of Genital Chlamydia}

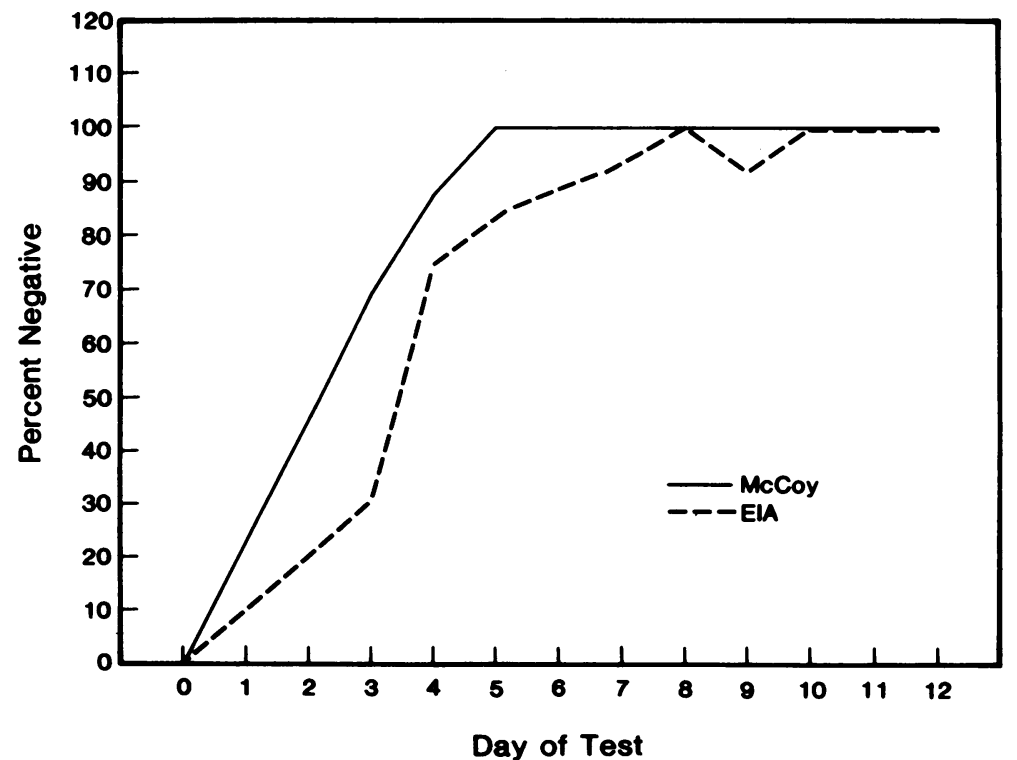


by culture five days after initiation of treatment. Non-viable organisms were not detected by ELISA ten days following initiation of therapy or three days following cessation of therapy. Thus, there appeared to be a five-day lag period of chlamydial antigen detection demonstrated by ELISA after culture (McCoy) confirmed cure (fig).

Both culture and EIA are reliable and may be used for tests of cure (TOC) if appropriate timing intervals are observed to avoid false positive TOC results. Since EIA was used by $50 \%$ of the United Kingdom clinics for TOC, the results of our investigation may be helpful. A reliable TOC can be obtained utilising EIA three days following completion of treatment (10 days post treatment initiation) instead of one week following treatment as reported by Radcliffe $e t$ al. Furthermore, other TOC indications may include pregnancy, complicated or severe infections, requests for psychological reassurance or for evidence of persistent cervicitis. However, as eloquently stated by Dr Radcliffe, a routine TOC for chlamydial endocervical infections is presently unessential.

\section{Department of Fin G FERRIS Medical College of Georgia, Augusta, Georgia 30912, USA}

1 Radcliffe KW, Rowen D, Mercey DE, et al. Is a test of cure necessary following treatment for cervical infection with Chlamydia trachomatis? Genitourin Med 1990;66:444-6.

2 Radcliffe KW, Rowen D, Mercey DE, et al. Survey of the management of Chlamydia trachomatis infection of the cervix. Genitourin Med 1991; 67:41-43.

3 Ferris DG, Lawler FH, Horner, et al. Test of cure for genital Chlamydia trachomatis infection in women. $J$ Fam Pract 1990;31:36-41.

\section{Syphilis and the elderly}

Dr B Chattopadhay' reports a case of a 91-year-old man found to have positive serological tests for syphilis prior to emigration. He states the difficulties encountered in accurate diagnosis.

Two geriatricians, a psychiatrist and myself ${ }^{2}$ in 1987 reviewed 659 consecutive medical and psychiatric admissions in the elderly to St James's Hospital, Leeds, where standard serological tests for syphilis were performed.

The ages ranged between 65 and 103 years (mean 81); 210 were men; 449 were women. Positive serological tests for syphilis were found in 23 $(3.5 \%)$ of the patients, whose ages ranged from 69 to 93 (mean 82.5). Seventeen were women and six were men. Even after extensive investigation $11(1.7 \%)$ could only be diagnosed as latent syphilis.

We were helped in diagnosis in that all the patients were white and a confidential venereal diseases treatment register exists in Leeds going back to 1919 in which we were able to check previous diagnoses.

MA WAUGH General Infirmary, Leeds, LSI 3EX, UK

1 Chattopadhay B. Syphilis and the elderly. Genitourin Med 1991;67: 270-1.

2 Corrado OJ, Bowie PCW, Bagnall WE, Waugh MA. The prevalence of positive serological tests for syphilis among elderly hospital patients. Age Ageing 1989;18:407-10.

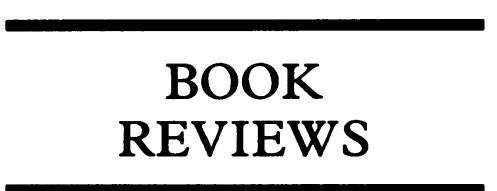

Genital Papillomavirus Infections. Modern Diagnosis and Treatment. Edited by $G$ Gross, S Jablonska, H Pfister, HE Stegner. (Pp 452; Price DM 148) Berlin. Springer-Verlag. ISBN 3-540-526153.

This monograph contains the documentation of The International Symposium on Genital Papillomavirus Infection held in Hamburg in 1989. It covers epidemiology, molecular biology, diagnosis, clinical aspects, immunological aspects, and therapy. The sixty-two contributors from ten countries are drawn from a wide range of specialities which deal with papillomavirus infection and the sequelae of such infection.

The sections on molecular biology and diagnosis are especially well presented. The authors ease the uninitiated painlessly into the world of papillomavirus biology. Terminology is explained such that non-specialist readers could readily follow the text without recourse to a dictionary of molecular biology.

The 11 chapters on clinical aspects economically cover a wide subject matter, and are generally well written. Illustrations, particularly the photomicrographs of cervical cytology were of a high quality. My only criticisms of this section concerned the DNA histograms which required further explanation for them to make their full impact, and that the chapter on vulval dystrophies seemed to be misplaced in this book, as papillomavirus is not mentioned at all.

The chapter entitled Immunology of Genital Papillomavirus Infections eloquently reviews humoral immunity, cell-mediated immunity, cellmediated cytotoxicity and local immune mechanisms. In a concise summary the relative importance of these individual components of the immune response to H.P.V., and the implications on future development of therapies is discussed.

I was a little disappointed not to find any information concerning loop excision diathermy in the section concerning therapy, although at the time of this conference this modality of treatment was just emerging. My disappointment was off-set by chapters on the basic science of cryotherapy and laser therapy.

Overall this book is well written and presented. It is not for the molecular biologist, but for the clinician with a interest in papillomavirus infection. The price may limit its sales but any clinician working in the field of such infections would, in my opinion, be advised to ensure that they have access to a copy in their clinics or libraries.

DAVID ROWEN

Economic Aspects of AIDS and HIV Infection. Edited by D Schwefel, R Leidl, J Rovira, M F Drummond (Pp 364, DM 148). Berlin: Springer-Verlag, 1990. 